\title{
Utilización didáctica de la historia de las ciencias: mujeres en ciencia nuclear
}

\author{
Mari Álvarez Lires* \\ Teresa Nuño Angós** \\ Uxío Pérez Rodriguez ${ }^{* * *}$
}

Resumen: En este artículo se parte de diversas contribuciones realizadas en el ámbito del análisis de la educación científica desde una perspectiva de género. Se hace un planteamiento general de la historia de las pioneras en ciencia nuclear $y$, en particular, se estudian un premio Nobel, Maria Göppert-Mayer (1906-1972) y otra brillante física, Wu Chien-Shiung (1912-1997), excluida de él. Finalmente se describen varias propuestas didácticas, susceptibles de usarse en el aula.

Palabras clave: Historia, ciencia, género, educación, pioneras, ciencia nuclear, propuestas didácticas.

\section{Didactic use of history of science: women in nuclear science}

Abstract: This article starts from the different contributions realized in the field of analysis of scientific education from a gender perspective. A general approach of the history of women pioneers in Nuclear Science is made. The article focuses particularly on the Nobel Prize Maria Göppert Mayer (1906-1972) and the prominent phisycist Wu ChienShiung (1912-1997), the latter was excluded from the prize. Finally, several didactic proposals capable of being used in the classroom are described.

Key words:History, science, gender, education, women pioneers, nuclear science, didactic proposal.

\footnotetext{
* Departamento de Didácticas Especiales. Universidad de Vigo. España.

** Departamento de Didáctica de las Ciencias Experimentales. Universidad del País Vasco. España.

*** Departamento de Didácticas Especiales. Universidad de Vigo. España.
}

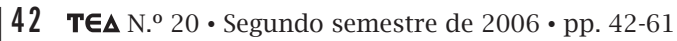




\section{Género, ciencia y educación}

Se ha afirmado, repetida y erróneamente, que las mujeres no han participado en la actividad tecnocientífica; para explicar este "hecho probado" se ha recurrido a lo largo de la historia a argumentaciones, supuestamente científicas, sobre una presunta incapacidad femenina para la abstracción o las destrezas requeridas en la actividad tecnológica.

En la década de los setenta se comenzó a utilizar el género como categoría de análisis en la investigación. Los Women Studies buscaron a las mujeres en los acontecimientos históricos y el papel desempeñado en ellos. Para Gerda Lerner (1986), las mujeres no han estado al margen sino en el centro de la formación de las civilizaciones, pues son la mitad de la humanidad y han compartido con los hombres el mundo y el trabajo. Han sido siempre sujetos históricos, aunque se les haya impedido interpretar y conocer la otra mitad de la historia, su historia.

A partir de los años ochenta, los Gender and Sciences Studies han revelado, además, que no sólo se ha impedido el acceso de las mujeres a la enseñanza oficial en cualquier momento histórico, sino que se las ha excluido de la tarea de elaborar sistemas simbólicos, filosofía, ciencias y leyes desde las instancias de poder. Se han realizado, pues, múltiples investigaciones para rescatarlas del ostracismo, devolverles su papel de sujetos históricos (Ogilvie, 1986; Alic, 1991; Álvarez-Lires, 1992; Solsona, 1992, 1996, 1997; Millar, 1997; A. Lires, Nuño y Solsona, 2003; A. Lires, 2006) y descubrir los rasgos androcéntricos presentes en la investigación e interpretación de datos (Keller, 1991; Sánchez, A., 1991), así como en la construcción y producción de la ciencia occidental moderna.

Las mujeres siempre han luchado contra su discriminación. No se han resignado al papel asignado; su acceso a la actividad científica no ha sido una excepción a pesar de que, como indica Sandra Harding (1996), se las ha excluido de la ciencia con más ardor que de los campos de batalla.

Con todo, si bien los tiempos han cambiado y las mujeres han podido acceder a la educación científica y trabajar en los centros tecnocientíficos actuales, su escasa representación en puestos relevantes ha preocupado a diversas instancias internacionales. El Informe Etan (2000) de la Unión Europea, European Technology Assessment Network on Women and Science, aportó datos incontestables sobre la posición de las mujeres en los ámbitos científico y tecnológico, demostrando que todavía se mantienen grandes obstáculos para una completa equidad de género y que, en consecuencia, no se está desarrollando en forma adecuada el potencial docente e investigador de las científicas. Por otra parte, en la Declaración de la Unesco sobre la Ciencia y el Uso del Conocimiento Científico (1998), se estableció lo siguiente:

La igualdad en el acceso a la ciencia no sólo es un requisito social y ético para el desarrollo humano, sino también una necesidad para la realización de todo el potencial de las comunidades científicas y para orientar el progreso científico hacia el conjunto de las necesidades de la humanidad. Las dificultades que encuentran las mujeres, que constituyen la mitad de la población mundial, para acceder y progresar en 
las carreras científicas, así como para participar en la toma de decisiones en ciencia y tecnología, deberían abordarse urgentemente.

\section{Educación científica}

Los esfuerzos hechos en las investigaciones sobre ciencia y género se correlacionan con estudios realizados en el campo educativo (Kelly, 1987; A. Lires, 1991; A. Lires et al., 1992, 1994a; A. Lires \& Soneira, 1994b; Nuño \& Ruipérez, 1997; Solsona, 1997). A continuación, se intentará ofrecer un breve resumen de estas contribuciones.

El acceso a la educación científica de las mujeres, su presencia mayoritaria en las facultades y escuelas universitarias -con excepción de las de ingeniería-, así como una importante participación en equipos de investigación, podrían hacer pensar en una situación de equilibrado entre hombres y mujeres en el campo de las ciencias experimentales. No obstante, un análisis riguroso indica que el problema, en las sociedades desarrolladas, no es ya el acceso de las mujeres a la educación y a la investigación, sino su escasa presencia en los centros de decisión, sus itinerarios profesionales y los efectos de la discriminación sobre su autoconcepto y sus expectativas de logro.

El análisis de género de las clases de ciencias proviene de los años sesenta en los países anglosajones, pero se ha de matizar que la mayor parte de las investigaciones se centraban en descubrir las supuestas carencias de las chicas en actitudes y aptitudes necesarias para dedicarse a la ciencia y a la tecnología, es decir, se partía del Paradigma de la debilidad (Catalá y García, 1989), que daba por descontado que sólo las actitudes y aptitudes de los chicos eran las precisas para ello. Con un enfoque semejante resulta muy difícil elevar la autoestima de las mujeres, que interiorizan que lo que han aprendido, como consecuencia de su socialización diferencial, es inútil para el sistema educativo. Las autoridades científicas, las imágenes y las actividades que se proponen tienen que ver, mayoritariamente, con intereses masculinos. Nada hay en la ciencia ni en la tecnología que haga referencia a la experiencia de las mujeres. Afortunadamente, en una parte de los estudios de género, epistemológicos y educativos, se ha pasado de cuestionar qué les ocurre a las mujeres que no acceden a la ciencia, y se pregunta qué le ocurre a la ciencia para que las mujeres no accedan a ella (Harding, 1996).

Así mismo, en diversos estudios se ha puesto de manifiesto que una parte del profesorado de ciencias experimentales, de todos los niveles educativos, se adscribe a una concepción de la ciencia que la considera una actividad lógica, neutral, objetiva, poseedora de verdades absolutas descubiertas por hombres geniales y desinteresados, con un estatuto de cuasiinfalibilidad, rasgos asociados a la conducta del varón blanco de clase mediaalta. La ciencia se presenta como una marcha triunfal de la verdad contra el error, de lo objetivo contra lo subjetivo, de lo fuerte contra lo débil... y, por ende, del triunfo de lo considerado masculino frente a aquello asociado a lo femenino. Esta concepción tiene consecuencias didácticas y, desde luego, dificulta el acceso de las mujeres a determinadas opciones profesionales y niveles de la investigación científica. Además, el sesgo masculino de la actividad científica no afecta sólo a las 
mujeres sino también a los hombres y a la propia ciencia (Keller, 1991).

En cualquier intervención didáctica existe un conjunto de concepciones implícitas que forman parte del currículo oculto, entre las que se encuentran el modelo de ciencia del profesorado y el que se presenta en los libros de texto. Si este modelo desmitifica su supuesta neutralidad y objetividad, y la contempla como una construcción personal y social enfatizando la responsabilidad de las comunidades científicas ante los problemas de la humanidad, puede ser más favorable a un enfoque coeducativo.

Actualmente, el sexismo en la enseñanza ya no se manifiesta en el acceso global a la educación ni en el rendimiento académico. Al menos en el Estado español, las alumnas son mejores estudiantes que los alumnos, circunstancia que se refleja en los resultados académicos y en el índice de abandono de los estudios, mucho más bajo que el de aquéllos. Pero esta situación académica no se traduce socialmente, ya que las mujeres siguen ocupando los puestos de trabajo menos valorados y su escasez en los centros de decisión es más que notable.

Es preciso señalar, además, que el sexismo limita la autonomía de las chicas y de los chicos, pues establece como referencia unos patrones rígidos femeninos y masculinos -estereotipos- a los que deben adaptarse para conseguir la aceptación social. Así por ejemplo, una chica puede expresar sus sentimientos, pero este comportamiento recibe la sanción grupal y social cuando quien los exterioriza es un chico. Vale la pena destacar que mientras que se admite socialmente la asunción de rasgos masculinos por parte de las mujeres, el estereotipo masculino es mucho más rígido, por lo que en los hombres no se permiten comportamientos considerados femeninos: los chicos no lloran, ni siquiera en el siglo XXI (Askew y Ross, 1991).

Esto significa que los roles y estereotipos limitan el desarrollo de la personalidad de hombres y mujeres pero, en la medida en que lo masculino está más valorado socialmente, las repercusiones para unas y otros son diferentes. Se podría afirmar que, en el caso de las mujeres, el sexismo existente dificulta su acceso al mundo público en condiciones de igualdad, limitando su autoestima, y en el caso de los varones impide o dificulta su acceso al mundo de lo privado y de los afectos. Puesto que el sexismo es un problema de desigualdad, asentado en prejuicios sociales e individuales, la actitud del profesorado es decisiva en el cambio de la situación descrita.

En los centros educativos procuran tratar a todo el mundo por igual, ya que niñas y niños ocupan las mismas aulas y su currículo es el mismo, pero olvidan que tratar realidades distintas de la misma forma es lo más discriminatorio que existe. En este caso significa, ni más ni menos, obligar a las niñas a integrarse en el patrón masculino que impregna la cultura occidental, sin tener en cuenta su socialización diferencial previa en intereses, habilidades, actitudes y aptitudes que, además, el sistema educativo desconsidera. Nada de lo que han hecho las mujeres en la historia de la humanidad se encuentra en los libros de texto ni en los contenidos que se transmiten y las figuras femeninas están ausentes, excepto casos como el de Marie Curie o el de la reina Isabel la Católica, por poner ejemplos sobradamente citados. 
Nada más incierto que esta ausencia femenina, pues aun suponiendo que las mujeres no hubiesen tenido nunca protagonismo en el mundo público, ¿es posible pensar que no hayan aportado nada valioso a lo largo de siglos de dedicación a la atención de las personas, a la alimentación, al vestido o a la salud de la familia? Tal suposición carece de soporte científico, a menos que se piense en las mujeres como seres inferiores; sin embargo, esa es la realidad de la cultura occidental que transmiten los manuales y el sistema educativo. La perspectiva de la historia, de la literatura y de la ciencia es únicamente masculina, de tal manera que toma al hombre como medida y patrón de todas las cosas, incurriendo en lo que se ha denominado androcentrismo que, en este caso, se manifiesta en la selección de los contenidos y en el tratamiento de la información en los materiales didácticos. Los saberes de las mujeres se desconsideran y se transmite, así, que solamente los hombres han sido protagonistas. Falta la mitad de la historia, pues resulta impensable que algún acontecimiento, histórico o científico, haya ocurrido sin presencia femenina.

Se han expuesto, pues, elementos de currículo oculto como la selección de contenidos, impregnada de androcentrismo, y la invisibilidad de las mujeres; sin embargo hay más, ya que en la intervención educativa, que dura muchos años, se evidencia la preponderancia del modelo masculino en el uso del lenguaje, que habitualmente olvida, margina o inferioriza a las mujeres mediante el uso abusivo del genérico masculino.
El sexismo se manifiesta también en la organización y dinámica de los centros, así como en el dualismo masculinofemenino, valorando el primer término del par y desconsiderando el segundo. Por ejemplo, se considera natural que, en aras de una supuesta libertad, los chicos manipulen aparatos y las chicas anoten, ordenen o limpien.

Una observación cuidadosa de las aulas indica que las interacciones entre el profesorado (masculino o femenino) y el conjunto de la clase se realizan de manera desequilibrada, puesto que el número de preguntas y respuestas dirigidas a los alumnos, así como el tiempo empleado, es mayor que cuando se dirigen a las alumnas, y todo ello ocurre de modo inconsciente. Cabe destacar la desigual ocupación de los espacios por parte de alumnas y alumnos, tanto del patio escolar como de los pasillos o de los laboratorios. Ellos ocupan, siempre, la mayor parte.

Desde la didáctica se ha señalado la necesidad de partir de las ideas y experiencias previas del alumnado para implicarlo en el control de sus aprendizajes pero, salvo que exista intención coeducativa explícita, ¿se tienen en cuenta las experiencias previas de las chicas o se considera que alguna de sus habilidades es útil para la ciencia escolar?

Igualmente, se sabe que ante un proceso de enseñanza no todo el alumnado progresa en la misma forma ni al mismo ritmo. Si bien el origen de las diferencias de aprendizaje es multicausal y depende de las capacidades personales, del origen social o de los aprendizajes previos realizados, pocas veces se toman en cuenta 
tales desigualdades, que pueden tener su origen en el género y, en consecuencia, no se incluyen orientaciones adecuadas en los planes de atención a la diversidad de los centros educativos.

No se debe olvidar que las diferencias dentro de cada colectivo de chicos y de chicas (intragrupos) son inmensas, de modo que no se debe generalizar: las chicas hacen [...], los chicos hacen [...] También hay alumnas que interrumpen y alumnos callados y trabajadores, pero las pautas de comportamiento generales indican importantes diferencias intergrupos.

Muchas alumnas manifiestan inseguridad a la hora de manipular instrumentos como la balanza, el microscopio, el amperímetro o el voltímetro. Tal circunstancia no se suele dar en los chicos, pero en cambio, es difícil que una alumna haga saltar los dispositivos de seguridad de un taller de electricidad, lo cual es bastante habitual en los alumnos varones; ellas acostumbran a preguntar antes de manipular, y si alguna intervención especial se debe hacer es animarlas a que hagan montajes y manejen los aparatos, obviamente con prudencia. Las alumnas suelen presentar los trabajos e informes de manera limpia y ordenada, y en la misma forma efectúan, por ejemplo, montajes eléctricos. Se observa, en cambio, que es difícil lograr que un elevado porcentaje de chicos realice un informe de manera ordenada y bien presentada, y la falta de cuidado en los montajes es bastante habitual.

Así pues, parece claro que se necesita una intervención diferenciada, sin perder de vista los estilos y ritmos de aprendizaje de cada persona, intervención que hoy reviste carácter de urgencia.

\section{Integración de la perspectiva de género}

Una integración de la perspectiva de género en el área de ciencias ha de permitir al alumnado caminar hacia la consecución, entre otros, de los siguientes objetivos:

- Adquirir una idea más abierta y menos dogmática de las ciencias.

- Comenzar a familiarizarse con un planteamiento interdisciplinar de las ciencias.

- Percibir que la historia de las ciencias y la de la humanidad no se han escrito separadamente, y que las mujeres han estado presentes en las dos.

- Identificar y valorar aportes femeninos a la ciencia, tecnología y bienestar.

- Cambiar de actitud respecto a la igualdad de mujeres y varones ante la ciencia.

- Mejorar la autoestima de chicas y chicos.

- Modificar el comportamiento agresivo e irreflexivo de un amplio colectivo de chicos.

- Superar las inseguridades manipulativas de un amplio colectivo de chicas.

- Aumentar el interés por las ciencias. De acuerdo con lo expuesto, un cambio necesario implica modificaciones en varios niveles. Siguiendo las orientaciones de diversos estudios de ciencia y género (Kelly, 1987; Keller, 1991; Askew y Ross, 1991; A. Lires et al., 1994a), señalaremos:

\section{a) Cambios en la producción de la ciencia}

Cambios orientados a la construcción de una ciencia que ha de convivir con la duda, el error y la incertidumbre, no 
poseedora de verdades absolutas, que intente convivir armónicamente con la naturaleza, abierta a la mirada interdisciplinar, que utilice el género como categoría de análisis. Estos cambios corresponden, fundamentalmente, a las comunidades científicas, pero el sistema educativo debe mostrarse favorable a ellos.

\section{b) Cambios en la ciencia escolar}

Una ciencia ligada al ámbito que nos rodea y a la historia, que tenga en cuenta la experiencia diferenciada de niñas y niños, y que considere las habilidades y destrezas "femeninas" como algo valioso para la propia ciencia y para cualquier persona, independientemente de su sexo; que recoja los aportes que las mujeres han hecho a lo largo de la historia; que tenga en cuenta la relación entre lo cognitivo y lo afectivo, y que incorpore las interacciones entre ciencia, tecnología, género y sociedad.

\section{c) Cambios en el profesorado}

El profesorado ha de adquirir instrumentos para detectar el sexismo y el androcentrismo en la ciencia y en la práctica escolar. Debe aprender a huir del pensamiento dicotómico y a incluir el género en sus categorías de análisis.

\section{d) Cambios en los chicos}

Se han de corregir los efectos no deseados de la construcción de la masculinidad, asociados al estereotipo de género: la competitividad, la agresividad, la desconsideración de los aspectos afectivos, así como el acaparamiento del material y de los espacios físicos y orales.

e) Cambios en las chicas

Se han de paliar los efectos no deseados de la construcción de la femineidad asociados al estereotipo de género, tales como la inseguridad y la desvalorización de lo propio. Un elemento indispensable en la acción coeducativa es el fomento de su autoestima.

\section{Estrategias para el cambio:} sugerencias para trabajar en el aula

Las estrategias que se pueden proponer para el cambio son múltiples y las hemos analizado en otros lugares (A. Lires et al., 1994; A. Lires et al., 2003); entre ellas se encuentran las que se examinan a continuación referentes al análisis y comentario de textos originales de científicas y al estudio y elaboración de sus biografías (Sánchez, M.D., 1999; Nuño, 2005).

En este caso se ha optado por estudiar la situación de las científicas nucleares que Nuño (2005) ha denominado "crónica de un olvido”. La utilización didáctica de las biografías de estas científicas y el análisis de sus textos sirven para enfatizar sus aspectos personales en cuanto mujeres de una época de la historia de la ciencia, así como sus contribuciones científicas y las conexiones entre sus descubrimientos. El análisis de sus aportes, en la identificación de nuevos elementos, al igual que sus investigaciones en ciencia nuclear, constituye un interesante caso histórico donde se ilustra el estratégico papel que han desempeñado en la creación de un área clave dentro de la ciencia contemporánea.

A continuación se proponen distintas actividades que se pueden utilizar en las clases de ciencias, para que el alumnado reflexione y discuta sobre diversos aspectos relacionados con la ciencia nuclear y las contribuciones hechas por científicas, así como sobre algunas de 
las discriminaciones y exclusiones que sufrieron por el hecho de ser mujeres.

\section{Redacción de una biografía}

En esta actividad se proponen pautas para analizar y la elaborar biografías de científicas. El informe o biografía se presentará oralmente, utilizando algún soporte visual (transparencias, póster, power point, etc.) que facilite su comprensión y resulte atractivo para su posible difusión y comunicación.

La propuesta de investigación se efectuará en horas lectivas pero la investigación en sí se hará en horario no lectivo, como trabajo monográfico. Por tanto, el seguimiento de éste se realizará en horas de tutoría o de consulta.

La investigación se plantea de la siguiente manera: se ofrecen una bibliografía básica de fácil acceso y una guía orientativa sobre los aspectos fundamentales que todas las biografías deben contener.

Con el fin de que el trabajo se realice de un modo cooperativo, cada estudiante deberá adjuntar al resumen final del grupo una copia de sus notas y aportes hechos durante el desarrollo de la investigación. La presentación no puede consistir en una lectura del mismo sino que, como ya se ha indicado, se utilizarán distintos recursos para ello. Cada estudiante deberá tomar notas durante las exposiciones, las cuales se recogeran y tomarán cuenta en la evaluación final.

Las biografías deberán constar, al menos, de los siguientes elementos básicos:

- Información personal: fecha y lugar de nacimiento y defunción, nombre y origen de la madre y del padre, clase social, religión, etnia,...
- Formación académica

- Investigación: formación investigadora, problemas científicos que estudió, desarrollo de sus investigaciones y contribuciones científicas.

- Experiencia profesional

- Habilidades profesionales

- Premios, nombramientos y distinciones

- Actividades, intereses y aficiones sociales y culturales, compromisos sociales y políticos.

- Breve descripción del contexto sociopolítico y de la situación de las mujeres en su época.

Cuestiones para la reflexión.

Analizar y discutir las siguientes cuestiones y añadir los resultados consensuados por el grupo al final de la biografía de la científica elegida:

- ¿Qué obstáculos tuvo que superar en su formación científica y para tener éxito o reconocimiento en el mundo científico?

- ¿Qué tipo de tratamiento recibe en los libros y manuales de ciencias? Comparado con el de los científicos de su época.

- ¿Qué tipo de prejuicios contra las mujeres, científicas y no científicas, ha encontrado al realizar la investigación? Quizá sea necesario contrastar sus opiniones con las de otros grupos para obtener un amplio abanico de ideas.

Esta experiencia podría completarse invitando a alguna científica, de la ciudad o de la universidad, para que explique su biografía. Con ello, se pretende dar al alumnado la oportunidad de conocer experiencias tangibles de mujeres profesionales del campo tecnocientífico. 


\section{Secuencia didáctica}

Se partirá de un planteamiento general de la historia de las pioneras en ciencia nuclear y, a continuación, se estudiarán las biografías y contribuciones científicas de una premio Nobel, Maria GöppertMayer (1906-1972), y de otra brillante física, Wu Chien-Shiung (1912-1997), excluida de él, o bien se elaborarán sus biografías tal como se ha indicado anteriormente.

\section{a) Pioneras en ciencia nuclear} (1897-1963)

En el siglo XX se incrementó notablemente la actividad científica de las mujeres gracias a que consiguieron acceder a la educación científica superior y participar en equipos de investigación, terrenos vetados para ellas anteriormente.
En cada uno de los descubrimientos del campo de la ciencia nuclear, desde finales del siglo XIX hasta mediados del siglo XX, las científicas estuvieron presentes. Algunas recibieron reconocimiento y prestigio en su época, y consiguieron el premio Nobel. Sin embargo, con excepción de Marie Sklodowska Curie (la única persona que ha conseguido dos premios Nobel -uno de física y otro de química- hasta el momento), pocas han llegado a formar parte de los libros de texto.

Además, no todas aquellas que merecieron el Nobel lo recibieron. Algunas quedaron eclipsadas por sus colegas varones, que sí lo obtuvieron. A continuación se incluyen dos cuadros, correspondientes a ambas categorías: las excluidas y las triunfadoras.

Científicas merecedoras del premio Nobel en física y química y científicos que los recibieron

\begin{tabular}{|c|c|c|c|}
\hline $\begin{array}{c}\text { Campo y año } \\
\text { del Nobel }\end{array}$ & $\begin{array}{c}\text { Científicas } \\
\text { (fechas de nacimiento } \\
\text { y defunción) }\end{array}$ & $\begin{array}{c}\text { Descubrimiento } \\
\text { y época de investigación }\end{array}$ & $\begin{array}{c}\text { Científicos premiados } \\
\text { (fechas de nacimiento y defunción) }\end{array}$ \\
\hline Química, 1944 & $\begin{array}{c}\text { Lise Meitner } \\
(1878-1968)\end{array}$ & $\begin{array}{c}\text { Fisión de núcleos pesados } \\
(1934-1939) .\end{array}$ & $\begin{array}{c}\text { Otto Hahn } \\
(1879-1968)\end{array}$ \\
\hline Física, 1957 & $\begin{array}{c}\text { Wu Chien-Shiung } \\
(1912-1997)\end{array}$ & $\begin{array}{c}\text { No conservación de la paridad en } \\
\text { interacciones nucleares débiles } \\
(1957) .\end{array}$ & $\begin{array}{c}\text { Tsung Dao Lee (1926-) } \\
\text { Chen Ning Yang (1922-) }\end{array}$ \\
\hline
\end{tabular}

Científicas premios Nobel en física y química

\begin{tabular}{|c|c|c|c|}
\hline $\begin{array}{c}\text { Época } \\
\text { descubrimiento }\end{array}$ & $\begin{array}{l}\text { Campo y año } \\
\text { del Nobel }\end{array}$ & Personas premiadas (Mujeres en cursiva) & Descubrimiento \\
\hline$(1897-1903)$ & $\begin{array}{c}\text { Física, } \\
1903\end{array}$ & $\begin{array}{l}\text { Marie Sklodowska Curie (1867-1934) } \\
\text { Pierre Curie (1859-1906) } \\
\text { Antoine Henri Becquerel (1852-1908) }\end{array}$ & La radiactividad y su interpretación. \\
\hline$(1903-1910)$ & $\begin{array}{l}\text { Química, } \\
1911\end{array}$ & Marie Sklodowska Curie (1867-1934) & $\begin{array}{l}\text { Descubrimiento del polonio y del radio y } \\
\text { aislamiento del radio. }\end{array}$ \\
\hline$(1931-1933)$ & $\begin{array}{l}\text { Química, } \\
1935\end{array}$ & $\begin{array}{l}\text { Irène Joliot-Curie } \\
(1897-1956) \\
\text { Frédéric Joliot-Curie (1900-1958) }\end{array}$ & $\begin{array}{l}\text { Radiactividad artificial. } \\
\text { Síntesis de nuevos elementos radiactivos. }\end{array}$ \\
\hline$(1948-1950)$ & $\begin{array}{c}\text { Física, } \\
1963\end{array}$ & $\begin{array}{c}\text { Maria Göppert-Mayer } \\
\text { (1906-1972) } \\
\text { J. Hans D. Jensen (1907-1973) } \\
\text { Eugene Paul Wigner } \\
\text { (1902-1995) }\end{array}$ & $\begin{array}{l}\text { Estructura del núcleo atómico (modelo de } \\
\text { capas). } \\
\text { Contribuciones a la teoría del núcleo atómico } \\
\text { y de las partículas elementales. }\end{array}$ \\
\hline
\end{tabular}


El trabajo pionero de Marie Sklodowska Curie (codescubridora y artífice de la interpretación de la radiactividad natural, y del polonio y del radio en 1898) continuó en Francia gracias a Irène Joliot-Curie (codescubridora de la radiactividad artificial y de la síntesis de nuevos elementos radiactivos en 1935) y en Alemania con Lise Meitner (codescubridora del protactinio en 1917 y de la fisión de núcleos pesados en 1939) e Ida Tacke Noddack (1896-1979) (codescubridora del Renio en 1925). Además, Ida Tacke Noddack, Irène Joliot-Curie y Lise Meitner estuvieron implicadas en el descubrimiento de la fisión nuclear.

Estos trabajos se completaron con otras contribuciones realizadas por Marguerite Perey (1909-1975) en Francia, que descubrió el francio en 1939, y por Berta Karlik (1904-1990) en Austria, que descubrió los isótopos del astato: $\mathrm{At}^{218,216,215}$. Por su parte, en Estados Unidos Maria Göppert-Mayer codescubrió el modelo de capas del núcleo atómico, en 1948, y Wu Chien-Shiung demostró empíricamente la noconservación de la paridad en las interacciones débiles entre partículas subatómicas, en 1957.

Como puede observarse en los cuadros anteriores, tres de estas científicas llegaron a recibir cuatro premios Nobel de física o química, y el trabajo de dos de ellas estuvo asociado a descubrimientos que contribuyeron a que sus compañeros de investigación recibieran dos premios Nobel, uno de química y otro de física.

Además, existe un tercer grupo de científicas, que trabajaron en los comienzos de la ciencia nuclear, en los laboratorios de los institutos de investigación sobre radiactividad más importantes de la época, entre quienes se destacan las siguientes:

- Fanny Cook Gates (1872-1931), Estados Unidos; Elisabeth R. Laird (18741969), Canadá;

- Harriet Brooks (1876-1933), Canadá. Ellen Gleditsch (1879-1968), Noruega.

- Ruth Pirret (?), Gran Bretaña

- Eva Ramstedt (1879-?), Edith Gertrude Willcock (1879-1953), Gran Bretaña; Jesse M. W. Slater (18791961), Inglaterra; Mary Sybil Leslie (1887-1937), Gran Bretaña; Jadwiga Szmidt (1889-1940), Polonia; Marietta Blau (1894-1970), Austria.

Todas ellas trabajaron, por lo general, con personajes de gran prestigio y reconocimiento, como:

- E. Rutherford (1871-1937), premio Nobel de química (1908).

- J.J. Thomson (1856-1940), premio Nobel de física (1906).

- Marie Sklodowska Curie (1867-1934), premio Nobel de física (1903) y de química (1911).

- Frederick Soddy (1877-1956), premio Nobel de química (1921).

- Bertram Boltwood (1870-1927).

No obstante, los trabajos de estas científicas no han tenido el reconocimiento que se merecían. A pesar de que existen numerosas publicaciones científicas de las que fueron autoras o coautoras, los libros de texto de física y química suelen atribuir la totalidad de los descubrimientos científicos de la época a sus coautores masculinos.

Por otro lado, cuando la comunidad científica ha reconocido, por sus extraordinarios aportes, a alguna científica, como por ejemplo Marie Curie, se ha producido un efecto pantalla o de 
ocultación sobre el trabajo y los éxitos de otras científicas coetáneas. Su papel como investigadoras noveles o en formación, además de ser representativo de la época, puede resultar atractivo a la mayoría del alumnado y especialmente a las alumnas, ya que muestra imágenes de otras científicas que son alternativas al modelo de Marie Curie, o complementarias con él.

La figura de Marie Curie ha sido tan utilizada como única representante del colectivo científico femenino, que podría provocar en las alumnas el denominado "síndrome Marie Curie" (Brush, 1985), según el cual, las jóvenes estudiantes creen que el éxito en ciencias requiere la devoción esclava al trabajo que ejemplifica la biografía de Curie y, ante esta perspectiva, muchas se alejan de la ciencia.

Los factores que propiciaron el acceso de mujeres a la investigación en la ciencia nuclear de principios del siglo $\mathrm{XX}$ fueron diversos. Por ejemplo, existen numerosas pruebas de que estas pioneras mantuvieron contactos personales y científicos entre sí, los cuales les aportaron, probablemente, el apoyo y estímulo que necesitaban para confirmar con su trabajo, en una época en que la sociedad no miraba con buenos ojos la incorporación de las mujeres a la ciencia y a otras esferas de la vida pública.

Otro de los factores que posibilitaron en algunos casos, y obstaculizaron, en otros, el acceso de mujeres a la investigación en ciencia nuclear fue el papel desempeñado por los grandes personajes del citado campo en esa época. Éstos se pueden clasificar en tres grupos:

- Las propias científicas del campo de la ciencia nuclear, en especial Marie
Sklodowska Curie, por el número de científicas colaboradoras que tuvo, fueron muy favorables a la incorporación de las mujeres a la ciencia. Podemos decir que se reconocieron y se dieron autoridad, sin esperar el reconocimiento de la autoridad masculina. Con ello sirvieron como modelo de identificación a las jóvenes estudiantes de ciencias del momento. Además, muchas de estas pioneras comenzaron sus investigaciones en ciencia nuclear antes de que Marie Curie obtuviera su gran fama y prestigio. Algunos científicos también se mostraron favorables a la admisión y contratación de científicas de distintos países en sus grupos de trabajo e investigación y, en algunos casos, a la causa de los derechos de las mujeres en general. En este apartado se destaca la figura de Ernest Rutherford.

- Aquellos científicos que, aunque contaron con mujeres en sus equipos de investigación, parecían tener una actitud tolerante, quizá demasiado condescendiente, e incluso paternalista, hacia ellas. Aquí podría incluirse a J.J. Thomson, B. Boltwood y F. Soddy, a juzgar por lo manifestado en su correspondencia. Thomson se casó con una de sus estudiantes de investigación, Rose Paget, que dejó su trabajo tras su compromiso matrimonial, y Soddy también contrajo nupcias con una de sus investigadoras, Winifred Moller Beilby.

- Los científicos que obstaculizaron el acceso de científicas a la investigación. Uno de ellos fue Emil Fischer (1852-1919), quien aceptó en su laboratorio a Lise Meitner después que ésta tuvo que prometerle que 
nunca entraría en él cuando hubiera hombres trabajando. Otro fue Max Planck (1858-1947) cuyas ideas contrarias a la educación científica de las mujeres eran conocidas en su época. Pero el principal enemigo de las científicas fue Otto Hahn, que ignoró por completo las contribuciones de Lise Meitner al descubrimiento de la fisión nuclear, tras haber mantenido con ella una estrecha colaboración científica durante 30 años. Además, rechazó valorar la propuesta que, cinco años antes del descubrimiento de la fisión nuclear, hizo Ida Tacke Noddack sobre la posibilidad de la fisión nuclear. Hahn escribió en sus memorias escribió al respecto: "Su sugerencia estaba tan fuera de lugar con las ideas sobre el núcleo atómico aceptadas entonces, que nunca se discutieron seriamente". Por último, a pesar de que Rutherford escribió a Hahn comunicándole que Harriet Brooks había encontrado previamente el fenómeno del retroceso del átomo radiactivo, tampoco quiso reconocerlo, a juzgar por el esfuerzo que hizo para refutarlo en sus memorias.

\section{b) Maria Göppert-Mayer (1906-1972)}

Nació en Katowice, Alta Silesia, que entonces pertenecía a Alemania. Fue hija única de Maria Wolff y Friedrich Göppert. Su padre, perteneciente a una antigua y culta familia de profesores universitarios, fue profesor de pediatría de la Universidad de Gotinga y director de un hospital infantil; además, fundó un centro para el cuidado de las criaturas de madres trabajadoras. Él inculcó a Maria su amor por la ciencia.
En esa época, los muchachos podían estudiar en las escuelas de Gotinga, pero las muchachas no. Maria pudo ingresar en un colegio privado, creado por un grupo de sufragistas con el fin de preparar a las chicas para los exámenes previos a la universidad. Pero en 1924 el colegio quebró a causa de la inflación y Maria tuvo que realizar el examen de bachillerato en Hannover. Sólo otras cuatro alumnas se presentaron con ella, junto a cientos de alumnos.

Ese mismo año ingresó en la Universidad de Gotinga para estudiar matemáticas y física. En 1927 se inclinó por la física, justo cuando, a través de las contribuciones de Max Born (18821970), Werner Heisenberg (1901-1976) y E. Pascal Jordan (1902-1980), estaba surgiendo en Götinga la nueva mecánica cuántica. En 1930 defendió su tesis doctoral sobre los cuantos ligeros, llamados fotones.

Maria, altamente calificada para las ciencias, no cumplía el arquetipo científico (dedicación única y exclusiva a la ciencia), ya que compaginaba su carrera investigadora con la vida social. Frecuentaba un círculo elegido de jóvenes académicos, futuras personalidades científicas, entre los que estaban el químico Linus Pauling (1901-1994), el físico Enrico Fermi (1901-1954), Eugene P. Wigner (1902-1995), J. Robert Oppenheimer (1904-1967), que dirigió el desarrollo de la bomba atómica, y Edward Teller (1908- ), "padre" de la bomba de hidrógeno.

En 1929, antes de acabar su tesis doctoral conoció a un químico californiano, Joseph E. Mayer, que había llegado a la Universidad de Gotinga para estudiar mecánica cuántica con el físico James 
Franck. En 1930, después de la lectura de la tesis de Maria, se casaron y se trasladaron a Estados Unidos. Al igual que Irène Curie y Frédéric Joliot, unieron sus apellidos en uno solo, compuesto.

Su marido había encontrado un trabajo académico remunerado en la destacada Universidad John Hopkins. Sin embargo, Maria, por ser mujer y esposa de un profesor universitario, no tenía oportunidad de encontrar empleo similar, pues además de la gran depresión económica, la ley contra el nepotismo prohibía la contratación simultánea de personas que fueran parientes o esposos. No obstante, ella continuó investigando en física sin remuneración y, con la influencia de su marido y de su colega Karl F. Herzfeld, se especializó en química-física. Trabajó en espectros moleculares utilizando métodos cuánticos y los tres publicaron varias investigaciones sobre colores y espectros de absorción de moléculas orgánicas.

En 1933 recibió la nacionalidad estadounidense y nació su primera hija, Marie Ann, que estudiaría astronomía, y en 1938 tuvo a su segundo hijo, Peter Conrad, que sería economista.

En 1939 se trasladaron a la Universidad de Columbia, en Nueva York, donde habían contratado a su esposo. De nuevo, Maria no pudo trabajar con la universidad, por lo que pasó un año como docente en el Sarah Lawrence College.

Investigó, junto con Harold C. Urey (1893-1981), la separación de isótopos del uranio y utilizó la teoría cuántica para calcular la posibilidad de existencia de un grupo de tierras raras de elementos transuránidos. Trabajó también con Edward Teller en una teoría del little- bang para explicar la abundancia de los elementos cósmicos.

Además publicó junto a su marido en 1940, un libro titulado Mecánica estadística y la contrató ese mismo año el Strategic Alloy Metals Laboratory. En este laboratorio se trabajaba, en secreto, en el marco del proyecto Manhattan, para el desarrollo de la bomba atómica.

Maria, que quería dedicar tiempo a sus hijos, ya que su marido permanecía ocupado en investigaciones militares, puso la condición de no trabajar los fines de semana ni cuando aquéllos estuvieran enfermos. Por este motivo parece que no se le asignaron proyectos importantes.

Después de la segunda guerra mundial (1939-1945), en 1946, la Universidad de Chicago llamó a Maria y Joe GöppertMayer, pero de nuevo Maria no percibió remuneración ni siquiera después de ascender a profesora numeraria, por lo que tuvo que compartir su investigación en el Instituto de Física Nuclear con otra remunerada en el Argonne National Laboratory (Laboratorio Nacional del Departamento de Energía Atómica de Estados Unidos).

Para Maria Göppert-Mayer, Chicago fue el primer lugar donde "no la contemplaban como una pesada carga, sino que se saludaban con los brazos abiertos". La física nuclear, que había florecido en la Universidad de Chicago durante la guerra con el fin de aplicarla e un modo práctico, continuó su auge en la posguerra, poniendo más atención en los hasta entonces descuidados problemas básicos (Fölsing, 1992).

En el Argonne National Laboratory se hicieron trabajos pioneros en el desarrollo de la utilización pacífica de la energía nuclear, como el de prototipos 
de reactores. En estos años, Maria volvería a encontrarse con viejos amigos como Enrico Fermi y Edward Teller, con quienes incrementó notablemente sus conocimientos de física nuclear.

En 1948, gracias a los modernos métodos matemáticos de la teoría de grupos, desarrolló un nuevo esquema de clasificación para los núcleos atómicos y sus propiedades más importantes. Encontró empíricamente el patrón de los "números mágicos": núcleos atómicos especialmente estables, con 2 (helio), 8 (oxígeno), 20 (calcio), 50 (estaño), 82 o 126 neutrones o protones.

Al principio, Maria no pudo ofrecer una explicación teórica a este descubrimiento empírico y postuló una analogía con la estructura de capas de los electrones en el átomo: el núcleo atómico consistía en protones y neutrones colocados en capas, igual que los electrones estaban dispuestos en el exterior del núcleo. En 1950, desarrolló el modelo de capas del núcleo atómico, en el que el acoplamiento spin-orbital predecía la ya observada estabilidad de los núcleos con números mágicos. En este modelo, los números mágicos describen núcleos en los que ciertas capas están completas con ese número de nucleones. Esta teoría hizo posible explicar, entre otros problemas, por qué algunos núcleos eran más estables que otros, el spin nuclear, los momentos magnéticos y por qué algunos elementos eran ricos en isótopos. Casi al tiempo, el físico alemán J. Hans Jensen (1907-1973) avanzó en la misma noción. En 1955, escribieron en colaboración un libro Teoría elemental de la estructura de capas nuclear, y un año después la eligieron miembro de la Academia de Ciencias de Estados Unidos.
En 1963, Maria Göppert-Mayer y Hans Jensen recibieron el premio Nobel de física por el modelo de capas del núcleo atómico, compartido con Eugene P. Wigner (1902-1995) por sus contribuciones a la teoría del núcleo atómico y de las partículas elementales. El Nobel se convirtió en el punto culminante de su carrera científica.

Las leyes predichas por Maria Göppert-Mayer y Hans Jensen se verificaron más tarde mediante observaciones y experimentos. El concepto teórico dio, con ello, un paso importante.

Antes del Nobel, en 1959, la Universidad de California en La Jolla, San Diego, ofreció al matrimonio Göppert-Mayer sendas plazas de titulares con salario de tiempo completo; así, Maria obtuvo por fin su primer puesto académico remunerado, como profesora de física.

Su salud, resentida desde 1960 a causa de un ataque de apoplejía cuyas secuelas le ocasionaron un defecto en el habla y una parálisis en el brazo izquierdo, comenzó a empeorar. Su corazón empezó a fallar y sufrió varios ataques, hasta que murió en 1972. Nunca abandonó la investigación y se comprometió cada vez más con la causa de la formación científica de las mujeres, alentando a las jóvenes a buscar su camino en las ciencias.

La Sociedad Americana de Física creó, en su honor, el premio Maria Göppert-Mayer, que se concede anualmente para destacar a mujeres relevantes en el campo de la física.

\section{b.1 El doble papel de Maria Göppert-Mayer}

A continuación se muestran las dificultades de una mujer para compaginar 
su trabajo de científica con su papel de madre.

En San Diego, Maria Göppert-Mayer se comprometió aún más con la defensa de los estudios científicos para las mujeres. En una conferencia que impartió en Japón, en 1965, titulada "La situación de cambio de la mujer vista por una científica”, dijo:

Las ciencias son realmente un campo excelente de estudio para las mujeres, especialmente la física o la química. Para mí, la física supone más diversión que cualquier otra materia. No hay ningún motivo para creer que las mujeres están en este aspecto menos capacitadas que los hombres, y que una mujer inteligente y bien cultivada no pueda aportar una contribución científica significativa.

Göppert-Mayer señalaba (GöppertMayer, 1965) las dificultades de una científica; sabía, por experiencia propia, que las mujeres que no quieren renunciar a marido e hijos se encuentran con una especial resistencia en el trabajo y también en la ciencia:

[...] Toda mujer quiere una vida completa, quiere casarse y tener hijos. Muchas mujeres solucionan este problema sacrificando todas sus actividades profesionales y viviendo sólo como esposas y madres. Eso es lo que hace ser reacios a algunos profesores de universidad a aceptar mujeres.

Previamente, había afirmado:

Desde siempre, desde que era una niña muy pequeña, he sabido qué se esperaba de mí cuando creciera, que adquiriera una educación o formación que me capacitara para ganarme mi medio de vida, de modo que no dependiera de un matrimonio
Ella advertía a las mujeres contra la renuncia de su trabajo:

No hay ningún motivo real para que una mujer casada abandone su carrera. Cuando se ve obligada a un par de años de inactividad, porque sus hijos son pequeños, al menos debería mantener contacto con su campo de estudio y seguir los nuevos descubrimientos y progresos en él. De esta manera, podrá retomar su carrera en cualquier momento.

Pero a ella misma no parecía resultarle muy fácil su doble papel de madre y mujer trabajadora:

Naturalmente, la combinación de niños y trabajo no es del todo fácil. Existe una presión emocional correspondiente a las lealtades enfrentadas a la ciencia, por un lado, y a los niños, por otro, que al fin y al cabo necesitan una madre. Yo he tenido esta experiencia completa. Pero cuando los niños se hacen mayores, entienden las relaciones y están orgullosos de tener a una científica por madre.

Pero niños formales y comprensivos no bastan: "Una científica casada necesita una pareja comprensiva. El marido adecuado para una mujer con una carrera de ciencia es un científico" (Fölsing, 1992).

A propósito del texto anterior y tras la lectura de la biografía de Maria Göppert-Mayer, además de estudiar y contextualizar sus aportes científicos, se pueden sugerir las siguientes cuestiones:

- ¿Qué obstáculos tuvo que superar, por ser mujer, Maria Göppert-Mayer tanto en su formación científica como para tener éxito o reconocimiento en el mundo científico? ¿Piensa que las ciencias son un campo excelente de 
estudio para las mujeres? ¿Y para los hombres?

- ¿Cree que las mujeres están igualmente capacitadas para la ciencia que los hombres? ¿Y para la tecnología?

- ¿La física o la química le parecen una diversión? ¿Por qué?

- ¿Cree que un hombre puede compaginar una vida pública, dedicada a la ciencia, con una vida privada con pareja sentimental o con criaturas? ¿Y una mujer? Argumente la respuesta.

- ¿Qué obstáculos o dificultades suelen encontrar las mujeres para acceder la ciencia y la tecnología y mantenerse en ella?

- ¿Está de acuerdo con la afirmación de Maria Göppert-Mayer cuando dice: "Toda mujer quiere una vida completa, quiere casarse y tener hijos"?

- ¿Cree que todo hombre quiere casarse y tener hijos?

- ¿Considera que una vida sin matrimonio o sin niños no puede ser completa? ¿Y una vida sin trabajo remunerado?

- ¿Cree, como afirma esta científica, que algunos profesores de facultades de ciencias y escuelas de ingeniería son reacios a aceptar mujeres por el interés de éstas en ser esposas y madres, o piensa que existen otras razones? ¿Cuáles?

- Dé su opinión sobre la siguiente afirmación de Maria: "Una científica necesita una pareja comprensiva. El marido adecuado para una mujer con una carrera de ciencia es un científico".

- Proponga soluciones o estrategias para que todas las personas, tanto mujeres como hombres, puedan compatibilizar la dedicación a su profesión con la dedicación a su familia.

\section{c) Wu Chien-Shiung (1912-1997)}

Nació en una pequeña ciudad cercana a Shanghai. Su madre, Fan Fuhua, era maestra de escuela y su padre, Wu Zhongy, dueño y director de la primera escuela femenina de su región. Su familia de ideas progresistas sobre la educación de las mujeres, dispuso que Wu estudiara hasta el cuarto grado en su escuela y, en 1922, que se trasladara a un pensionado de Suzhou, donde finalizó sus estudios secundarios en la especialidad de maestra, con el número uno de su promoción. Cabe recordar que, en esa época, en algunas zonas de China todavía se vendaban los pies a las niñas, impidiendo su desarrollo y crecimiento normales.

En 1930, ingresó a la prestigiosa Universidad Nacional Central de Nankín, donde desarrolló una gran actividad en el movimiento estudiantil. En 1934, tras su graduación en física, trabajó un año como profesora en una universidad provincial e investigó, durante otro año, sobre cristalografía de rayos X en la Academia de Ciencias de Shanghai.

En 1936 se trasladó a Estados Unidos, donde la admitieron, como doctoranda, en el equipo de investigación del Laboratorio de Radiación de la Universidad de California, dirigido por el físico estadounidense Ernest Lawrence (1901-1958), inventor del ciclotrón (acelerador de partículas). Wu se doctoró en 1940.

La física parece haber sido lo más importante en la vida de $\mathrm{Wu}$, como lo confesaba abiertamente: "Siempre he tenido la sensación de que debía dedicarme a la física totalmente y sin limitaciones. 
No es un trabajo. Es una forma de vivir" (McGrayne, 1998).

En 1942 se casó con el físico chino Luke Chia-Liu Yuan, con quien tendría un hijo, Vincent, que también sería físico. Trabajó como profesora ayudante en el Smith College y en 1943 llegó a ser la primera mujer instructora de física de la Universidad de Princeton.

De 1944 a 1946 trabajó en el Proyecto Manhattan, en la construcción de la bomba atómica. A partir de 1946, fue investigadora asociada en la Universidad de Columbia y en 1952 pasó a ser profesora asociada de esta Universidad.

Durante estos años volvió a ocuparse de un tema popular en física nuclear, que ya había abordado en su tesis doctoral: la desintegración $\beta$ en los átomos radiactivos. Los experimentos físicos de la época sólo habían podido encontrar electrones de bajo movimiento en las desintegraciones $\beta$.

Wu detectó un fallo experimental y diseñó una serie de experimentos precisos y controlados para investigar los electrones que atravesaban láminas de espesor uniforme y encontró, tal como Fermi había predicho, que los electrones se movían a gran velocidad. Los experimentos realizados por $\mathrm{Wu}$ supusieron la confirmación experimental de algunas teorías, que se habían aceptado en física nuclear sin evidencia empírica, y le aportaron un gran prestigio en física experimental, destacando su exactitud y atención a los detalles.

T.D. Lee (1926- ) y C.N. Yang (1922- ) recibieron el premio Nobel de física en 1957 por su hipótesis de la no conservación de la paridad en las interacciones débiles entre partículas subatómicas, convirtiéndose así en los primeros cien- tíficos chinos en obtener tal galardón. Pese a que el diseño y la confirmación experimental de dicha hipótesis teórica los había aportado Wu, a ella y a los investigadores (E. Ambler, R.P. Hudson, R.W. Hayward y D.D. Hoppes) que le colaboraron en la realización práctica del experimento los excluyeron del Nobel.

De nuevo, se despojaba a una mujer de su autoridad científica y sus contribuciones quedaban enmascaradas bajo los aportes complementarios de sus colegas hombres. Sin embargo, Wu recibió muchos otros premios y reconocimientos científicos. Entre 1958 y 1975 le concedieron once doctorados honoris causa, entre los que cabe destacar los de las universidades de Yale, Harvard y Princenton, siendo este último el primero otorgado a una mujer. Fue miembro de la Academia de Ciencias de China y de la de Estados Unidos y se convirtió en la primera mujer presidenta de la Sociedad Americana de Física. Recibió también la Medalla Nacional de la Ciencia estadounidense.

En 1958 la ascendieron a profesora titular de física en la Universidad de Columbia.

En 1963 confirmó experimentalmente la ley de la conservación del vector corriente en la desintegración $\beta$, que habían propuesto R. Feynman y M. GellMann. Esto hizo avanzar el desarrollo de la actual teoría unificada de las fuerzas fundamentales.

Además, Wu aplicó a la medicina su trabajo en física nuclear. Desarrolló un método de estudio de las células enfermas en la anemia mediante técnicas de física nuclear.

En 1972 llegó a ser catedrática de física de la Universidad de Columbia. 
Allí continuó su docencia e investigación en física nuclear hasta su jubilación, en 1981.

De 1975 a 1982 fue miembro del Advisory Committee to the Director de los Institutos Nacionales de Salud.

También se preocupó especialmente por el papel de las mujeres en la sociedad y en las ciencias. Después de su jubilación, dio numerosas conferencias en las que animaba a las jóvenes a elegir carreras científicas.

Como esposa, madre y científica desempeñó los múltiples roles con que se enfrentan las mujeres en la sociedad moderna. Afirmaba que su deseo de ser buenas compañeras y buenas madres es deseo noble, pero que lo deben los hombres, y que el modo más equilibrado de criar y educar a una criatura es con los cuidados del padre y de la madre.

\section{c.1 Contribuciones de Wu Chien-Shiung a la ciencia nuclear}

Después de leer la biografía de Wu se puede proponer la realización de un estudio, contextualización y resumen de sus contribuciones a la ciencia nuclear y plantear las siguientes cuestiones:

- ¿Conoce un libro de Isaac Asimov titulado El electrón es zurdo, publicado por la Editorial Alianza en 1977? Busque información sobre él y el porqué de su título.

- ¿Por qué el Nobel de física de 1957 fue sólo para Lee y Yang?

- En la producción de conocimientos científicos, ¿cree que es más importante el planteamiento del marco teórico y de las hipótesis que el diseño experimental creativo y su realización práctica rigurosa? Argumente la respuesta.
- ¿Sabe cuál es el número máximo de personas que puede compartir un premio Nobel?

- Proponga un reparto más justo del Nobel de física de 1957. Tras la puesta en común de las decisiones de los distintos grupos, júntense quienes hayan apoyado la misma candidatura y esgriman argumentos que apoyen su elección.

- ¿Qué obstáculos tuvo que superar Wu a lo largo de su vida, y gracias a qué estrategias o ventajas pudo superarlos?

- Manifieste su acuerdo o desacuerdo con las opiniones de Wu sobre los deseos de las mujeres y la mejor forma de criar y educar a los hijos.

c.2 Opiniones de Wu Chien-Shiung sobre el tipo de obstáculos que impiden la participación de mujeres en ciencia y tecnología

En el siguiente texto se recogen algunas opiniones de Wu Chien-Shiung sobre la dificultad que encuentran las mujeres para participar en la empresa científica:

"Yo dudo sinceramente de que cualquier persona libre de prejuicios crea en verdad en la errónea idea de que las mujeres no tienen capacidad intelectual para la ciencia y la tecnología. Tampoco creo que los factores sociales y económicos sean los obstáculos reales que impiden la participación de las mujeres en el campo científico y técnico.

[...] El principal obstáculo en el camino de cualquier tipo de progreso es y ha sido siempre la irrecusable tradición" (McGrayne, 1998).

Tras la lectura del texto anterior se pueden proponer al alumnado las siguientes cuestiones: 
- Manifieste su acuerdo o desacuerdo con las opiniones de Wu recogidas en el texto anterior.

- ¿Cree que la tradición es lo que obstaculiza el acceso y la permanencia de las mujeres en la ciencia y la tecnología?

- ¿Qué otros factores contribuyen al hecho de que muchas mujeres se alejen de la ciencia y de la técnica?

- ¿Cree que las mujeres tienen menor capacidad intelectual que los hombres para la ciencia y la tecnología?

\section{Bibliografía}

Alic, M. (1991). El legado de Hipatia. Madrid: Siglo XXI Editores.

Álvarez-Lires, M. (1991). Ciencias experimentales. ¿Carencias de las chicas? La enseñanza de las matemáticas y las ciencias experimentales. III Jornadas Internacionales de Coeducación. Valencia: Institut Valencià de la Dona.

Á. Lires, M. et al. (1992). Propuestas para una enseñanza no sexista de ciencias naturales, física y química, matemáticas e informática, Hacia una escuela coeducadora. Posgrado de Coeducación. Vitoria-Gasteiz. Universidad del País Vasco-Emakunde.

Á. Lires, M. et al. (1994a). Materiales curriculares para la ESO. Ciencias de la naturaleza. Un enfoque coeducativo desde la historia de las ciencias en Occidente. Sevilla: Junta de Andalucía. Consejería de Educación y Ciencia.

Álvarez-Lires, M. y Soneira, G. (1994 b). Enseñanza y aprendizaje de las ciencias experimentales: la coeducación como meta. Premios Cide-Mec 1992. Madrid: Ministerio de Educación y Ciencia.
- ¿Piensa que la ciencia y la técnica son cosas de hombres? Argumente la respuesta.

\section{A modo de conclusión}

De acuerdo con la experiencia, la utilización de las actividades didácticas propuestas contribuye a que el alumnado adquiera un mayor interés por la historia de la ciencia y por el tema objeto de estudio; en este caso, la ciencia nuclear y la participación de las mujeres en ella. $\boldsymbol{\Delta}$

Álvarez-Lires, M.; Nuño, T. y Solsona, N. (2003). Las científicas y su historia en el aula. Madrid: Síntesis.

Álvarez-Lires, M. (2006). La educación científica de las mujeres en el siglo XVII: sor Juana Inés de la Cruz (México, 1648-1695). Revista de Investigación en Educación, 2, 175-212.

Askew, S. y Ross, C. (1991). Los chicos no lloran. Barcelona: Paidós Educador.

Brush, S. (1985). Women in physical science: From drudges to discoverers. Physics Teacher, 23, 11-19.

Catalá, E. y García, A. (1989). ¿Qué quieres hacer de mayor?, o la transición desde la coeducación. Valencia: Generalitat Valenciana.

Etan (2000). European Technology Assessment Network on Women and Science. Expert Working Group on Women and Science: Science policies in the European Union. Bruselas: European Commission, Research Directorate General.

Fölsing, U. (1992). Mujeres premios Nobel. Madrid: Alianza. 
Göppert-Mayer, M. (1965). The changing status of women as seen by a scientist. San Diego: Universidad de California.

Harding, S. (1996). Ciencia y feminismo. Madrid: Morata.

Keller, E. F. (1991). Reflexiones sobre género y ciencia. Valencia: Alfons el Magnànim.

Kelly, A. (1987). Science for girls? Filadelfia: Open University Press.

McGrayne, S. B. (1998). Nobel Prize women in science. Their lives, Struggles and momentous discoveries. Secaucus, N. J.: Carol Publishing Group Edition.

Millar, D.; Millar, I.; Millar, J. y Millar, M. (1996). The Cambridge dictionary of scientists. Cambridge: Cambridge: University Press.

Nuño, T. (2005). Pioneras en ciencia nuclear (1897-1963). Crónica de un olvido. Bilbao: Universidad del País Vasco/EHU.

Nuño, T. y Ruipérez, T. (1997).Análisis de los libros de texto desde una perspectiva de género. Alambique, $11,55-64$.

Ogilvie, M. B. (1986). Women in science. Cambridge, Massachusetts. Londres: The MIT Press.
Rayner-Canham, M. F. y Rayner-Canham, G.W. (1990). Pioneer women in nuclear science. Am. J. Phys., 58, 1036-1043.

Sánchez, A. (1991). La masculinidad en el discurso científico: aspectos epistémico-ideológicos, En: L. Luna (ed.), Mujeres y sociedad. Nuevos enfoques teóricos y metodológicos. Seminario Interdisciplinar Mujeres y Sociedad. Universitat de Barcelona.

Sánchez, M. D. (1999). Las biografías de las científicas en la enseñanza de las ciencias: mostrando una tradición", En: M. J. Barral, C. Magallón, C. Miqueo y M. D. Sánchez, Interacciones ciencia y género. Icaria-Barcelona: Antrazyt.

Solsona, N. (1992). La contribució de les dones a la història de la ciència. Santa. Coloma de Gramenet: Casal del Mestre.

Solsona, N. (1996). La voz de las mujeres en la ciencia de los siglos XVII y XVIII. Ingenium, 5, 125-136.

Solsona, N. (1997). Mujeres científicas de todos los tiempos. Madrid: Talasa.

Unesco (1998). European Regional Conference on Women in Science-Quality and Equality. Conditions for Sustainable Human Development. Eslovenia: Bled. 